\title{
GAD antibodies can signal downbeat nystagmus
}

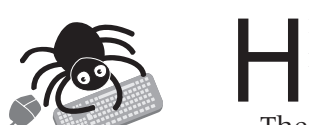

igh titre glutamic acid decarboxylase (GAD) antibodies may be a reason for downbeat nystagmus (DBN), say neurologists reporting on a single case. This is the first report of a link with GAD antibodies; DBN is often associated with lesions in the brain.

The 66 year old woman presented with three months' progressive oscillopsia, blurred vision, and vertigo. She had spontaneous vertical DBN in the primary position and on lateral gaze, not inhibited by fixation, and unsteady gait veering to the right. There were no signs of neurological conditions such as stiff man syndrome (SMS) or palatal myoclonus, or of brain lesions on imaging.

GAD antibodies were present at high titre $(>100 \mathrm{U} / \mathrm{ml})$ in her serum and cerebrospinal fluid, initially and eight months later, when her symptoms were worse. They were specific for native $\mathrm{GAD}_{65}$ and reacted strongly against recombinant GAD amino acid fragment 290585 but not fragment 290-511. They also occurred in serum from patients with SMS and palatal myoclonus included for comparison and reacted similarly. Control samples from 44 blood donors were negative.

The woman underwent exhaustive tests to rule out other diagnoses. GAD antibody was detected by immunostaining and confirmed by immunoprecipitation assay of recombinant human $\mathrm{GAD}_{65}$.

GAD converts glutamic acid to $\gamma$-aminobutyric acid (GABA) and is located in pancreatic $\beta$ cells and GABA secreting neurones throughout the CNS. GAD antibodies occur in greater proportion in SMS and have been reported in subacute cerebellar ataxia, epilepsy, and palatal myoclonus. The cause of DBN is unknown in $40 \%$ of cases.

A Journal of Neurology, Neurosurgery, and Psychiatry 2003;74:998-999. 